\title{
PROCESSO SALUTE-MALATTIA IN AMAZZONIA: FATTORI AMBIENTALI E COMPARSA DI MALATTIE
}

\section{REVISIONE ARTICOLO}

CUSTÓDIO, Wenderson Picanço, CHAVES, Juliana Vitória Rocha Leite, PANTOJA, Patrícia Trindade, CÁRDENAS, Anneli Mercedes Celis de, ANDRADE, Rosemary Ferreira de, DAPUREZA, Demilto Yamaguchi, FECURY, Amanda Alves

CUSTÓDIO, Wenderson Picanço. Et al. Processo salute-malattia in Amazzonia: fattori ambientali e comparsa di malattie. Revista Científica Multidisciplinar Núcleo do Conhecimento. Anno. 06, Ed. 12, Vol. 03, pp. 05-21. Dicembre 2021. ISSN: 24480959, Link di accesso: https://www.nucleodoconhecimento.com.br/salute/comparsadi-malattie, DOI: 10.32749/nucleodoconhecimento.com.br/salute/comparsa-dimalattie

\section{RIEPILOGO}

Introduzione: L'Amazzonia è sempre stata oggetto di numerosi studi incentrati sul suo ambiente, la società e la salute. Domanda guida: Quali fattori ambientali contribuiscono all'emergere di malattie nella regione amazzonica? Obiettivo: Affrontare i principali fattori ambientali che contribuiscono all'emergere di malattie nella regione amazzonica. Metodo: Questa è una revisione della letteratura nei database Scientific Electronic Library Online (SciELO), US National Library of Medicine National Institutes of Health (PubMeD), Biblioteca Virtuale della Salute (BVS) e National Institute of Space Research (INPE). In tutto, 18 articoli sono stati utilizzati nella costruzione dello studio, tutti passati attraverso i criteri di inclusione ed esclusione stabiliti al fine di filtrare solo le riviste con approcci tematici. Risultati: Dal punto di vista epidemiologico, lo spazio amazzonico formato dall'interazione del sistema socio ecologico ha caratteristiche diverse da altre parti del paese. Questa differenza è causata dal fondamento ecologico naturale e dalle sue forme di occupazione e sviluppo. Conclusione: i principali fattori ambientali che contribuiscono all'emergere di 
malattie nella regione amazzonica sono la deforestazione, la combustione, l'inquinamento dei fiumi, il processo di urbanizzazione e l'agribusiness.

Parole chiave: Salute-malattia, Amazzonia, Fattori ambientali.

\section{INTRODUZIONE}

L'Amazzonia è sempre stata oggetto di numerosi studi incentrati sul suo ambiente, società e sviluppo. Le pubblicazioni degli ultimi decenni hanno discusso importanti aspetti regionali come la biodiversità, gli usi del suolo, la_scienza e la tecnologia, la crescita urbana (BROWDER e GODFREY, 2017), lo sviluppo sostenibile e i progetti di sviluppo che causano la deforestazione (PERZ, 2002).

Per comprendere la complessità dell'Amazzonia in relazione alle diverse regioni del mondo, è importante ricordare che questa complessità non si limita alle sue caratteristiche biologiche e ambientali, ma è correlata al modo in cui la popolazione che la abita differisce nel suo modo di vivere e nel modo di agire e pensare (ROLIM, 2015).

II concetto di malattia della salute può assumere una dimensione regionale, poiché in questa regione c'è un immenso vuoto di informazioni scientifiche sulle condizioni sociali e ambientali, per soddisfare le esigenze della società. Vale la pena ricordare che in questa regione c'è un'alta concentrazione di persone fissate nei centri urbani, specialmente nelle capitali, causando problemi diffusi nei grandi centri come: mancanza di acqua trattata e servizi igienico-sanitari di base, proliferazione di insetti vettori di malattie, malaria che trasmette no usualmente anofelina, dengue tra gli altri (CAÑETE e RAVENA-CAÑETE, 2010).

La scelta di questa regione come oggetto di analisi è giustificata dal suo aspetto ambientale (clima, ecosistemi e biodiversità) e demografico (diversità sociale con bassa densità di popolazione e concentrazione nelle città), che, in combinazione con processi economici come l'agricoltura estensiva, l'allevamento, lo sfruttamento minerario, l'energia idroelettrica e l'industria hanno un impatto sulla salute della popolazione secondo il suo modello di sviluppo. Sulla base di questo, la domanda RC: 104517

Link di accesso: https://www.nucleodoconhecimento.com.br/salute/comparsa-di-malattie 
principale dello studio è: quali fattori ambientali contribuiscono all'emergere di malattie nella regione amazzonica?

Pertanto, lo scopo di questo studio era quello di evidenziare i fattori ambientali che contribuiscono all'emergere di malattie nella regione amazzonica, nonché di enfatizzare la ricerca diretta alla regione, consentendo una più ampia raccolta bibliografica sul tema, oltre a evidenziare la necessità di creare e riformulare politiche di salute pubblica per la popolazione che vive in questa regione.

\section{METODOLOGIA}

Questo lavoro è stato svolto attraverso una revisione della letteratura su riviste scientifiche, disponibili sui siti web e sui database: Scielo, pubmed, Virtual Health Library e studi condotti dall'istituto nazionale di ricerca spaziale. Gli articoli sono stati cercati tra agosto e novembre 2021 e sono stati raccolti 18 articoli tutti selezionati per la costruzione dello studio. I criteri di inclusione erano articoli pubblicati dal 2000 al 2021, in inglese, spagnolo e portoghese. D'altra parte, i criteri di esclusione erano articoli pubblicati prima dell'anno 2000, duplicati, editoriali della lettera dell'editore, nonché riviste in lingue diverse da quelle menzionate nei criteri di inclusione. I descrittori utilizzati per cercare gli studi erano: Salute-malattia; Amazzonia; Fattori ambientali.

Pertanto, questo articolo è un'analisi del processo di malattia sanitaria nella regione amazzonica e dei fattori ambientali che influenzano l'emergere di malattie (fattori sociali, economici, culturali e ambientali e altri fattori che influenzano il verificarsi di problemi di salute nella popolazione), Secondo Sobral e Freitas (2010) utilizzando variabili quantificabili (indicatori) e revisione della letteratura.

\section{RISULTATI}

Tabella 1. I migliori risultati di articoli selezionati per la ricerca

Titolo

\section{RC: 104517}

Link di accesso: https://www.nucleodoconhecimento.com.br/salute/comparsa-di-malattie

\section{Autori, anno di Principali risultati pubblicazione}




\begin{tabular}{|c|c|c|c|}
\hline & & e paese & \\
\hline $\begin{array}{l}\text { ARTICOLO } \\
\text { I }\end{array}$ & $\begin{array}{l}\text { A evolução do } \\
\text { conhecimento sobre } \\
\text { hepatites virais na } \\
\text { região Amazônica: da } \\
\text { epidemiologia } \quad \text { e } \\
\text { etiologia } \\
\text { prevenção. }\end{array}$ & $\begin{array}{l}\text { Bensabath e } \\
\text { Soares, 2016, } \\
\text { Brasil. }\end{array}$ & $\begin{array}{l}\text { Lo studio cerca di } \\
\text { dimostrare una malattia } \\
\text { simile alla febbre gialla, ma } \\
\text { considerata come una } \\
\text { nuova malattia, si verifica } \\
\text { nelle aree delle valli dei } \\
\text { fiumi Juruá, Purus e } \\
\text { Madeira. Temuto dai } \\
\text { residenti per l'alta letalità, } \\
\text { essendo clinicamente un } \\
\text { epatoencefalopatia di } \\
\text { evoluzione fulminante } \\
\text { (media di 5-6 giorni). }\end{array}$ \\
\hline $\begin{array}{l}\text { ARTICOLO } \\
\text { II }\end{array}$ & $\begin{array}{l}\text { Chagas disease and } \\
\text { globalization of the } \\
\text { Amazon. }\end{array}$ & $\begin{array}{l}\text { Briceño-Léon, } \\
\text { 2007, Brasil. }\end{array}$ & $\begin{array}{l}\text { L'articolo in questione } \\
\text { solleva un aumento del } \\
\text { numero di casi autoctoni di } \\
\text { malattia di Chagas in } \\
\text { Amazzonia dal 1970, } \\
\text { portando a temere che la } \\
\text { malattia diventi un nuovo } \\
\text { problema di salute pubblica } \\
\text { nella regione.Questa } \\
\text { trasformazione nel modello } \\
\text { epidemiologico della } \\
\text { malattia in Amazzonia può } \\
\text { essere spiegata dai } \\
\text { cambiamenti ambientali e } \\
\text { sociali degli ultimi } 30 \text { anni. }\end{array}$ \\
\hline $\begin{array}{l}\text { ARTICOLO } \\
\text { III }\end{array}$ & $\begin{array}{ll}\text { Rainforest } & \text { Cities. } \\
\text { Urbanization, } & \\
\text { Development } & \text { and }\end{array}$ & $\begin{array}{l}\text { Browder } \quad \text { e } \\
\text { Godfrey, 2017, } \\
\text { Estados }\end{array}$ & $\begin{array}{l}\text { Lo studio ha dimostrato la } \\
\text { riconciliazione delle } \\
\text { questioni ambientali di }\end{array}$ \\
\hline
\end{tabular}

RC: 104517

Link di accesso: https://www.nucleodoconhecimento.com.br/salute/comparsa-di-malattie 


\begin{tabular}{|c|c|c|c|}
\hline & $\begin{array}{l}\text { Colonization of the } \\
\text { Brazilian Amazon. }\end{array}$ & Unidos. & $\begin{array}{lr}\text { fronte } & \text { all'attuale } \\
\text { urbanizzazione } & \text { globale } \\
\text { considerando l'intensa } \\
\text { transizione urbana in corso } \\
\text { nella regione amazzonica. }\end{array}$ \\
\hline $\begin{array}{l}\text { ARTICOLO } \\
\text { IV }\end{array}$ & $\begin{array}{l}\text { Populações } \\
\text { Tradicionais } \\
\text { Amazônicas: } \\
\text { revisando conceitos. }\end{array}$ & $\begin{array}{l}\text { Cañete } \quad \text { e } \\
\text { Ravena- } \\
\text { Cañete, 2010, } \\
\text { Brasil. }\end{array}$ & $\begin{array}{l}\text { Il lavoro mirava a discutere } \\
\text { criticamente il concetto di } \\
\text { popolazione tradizionale } \\
\text { all'interno del contesto } \\
\text { amazzonico, verificando } \\
\text { che il concetto di } \\
\text { popolazione tradizionale, } \\
\text { inteso come identità } \\
\text { collettiva che ha la } \\
\text { specificità di sviluppare } \\
\text { modi di vita di integrazione } \\
\text { e intimità con l'ambiente. }\end{array}$ \\
\hline $\begin{array}{l}\text { ARTICOLO } \\
\text { v }\end{array}$ & $\begin{array}{l}\text { O trabalho como } \\
\text { determinante do } \\
\text { processo saúde- } \\
\text { doença. }\end{array}$ & $\begin{array}{l}\text { Cardoso, 2015, } \\
\text { Brasil. }\end{array}$ & $\begin{array}{l}\text { Lo studio ha analizzato } \\
\text { come, nel contesto attuale, } \\
\text { le condizioni organizzative } \\
\text { e fisiche del lavoro, le } \\
\text { situazioni e i rapporti di } \\
\text { lavoro e le forme digestione } \\
\text { stiano determinando e } \\
\text { contribuendo alla malattia } \\
\text { dei lavoratori per } \\
\text { comprendere una relazione } \\
\text { così complessa. }\end{array}$ \\
\hline $\begin{array}{l}\text { ARTICOLO } \\
\text { VI }\end{array}$ & $\begin{array}{l}\text { Determinante da } \\
\text { saúde no Brasil: a } \\
\text { procura da equidade } \\
\text { na saúde. }\end{array}$ & $\begin{array}{l}\text { Carrapato et } \\
\text { al., } 2017, \\
\text { Brasil. }\end{array}$ & $\begin{array}{l}\text { Il presente studio mirava ad } \\
\text { individuare, attraverso la } \\
\text { bibliometria, quali sono i } \\
\text { determinanti della salute }\end{array}$ \\
\hline
\end{tabular}




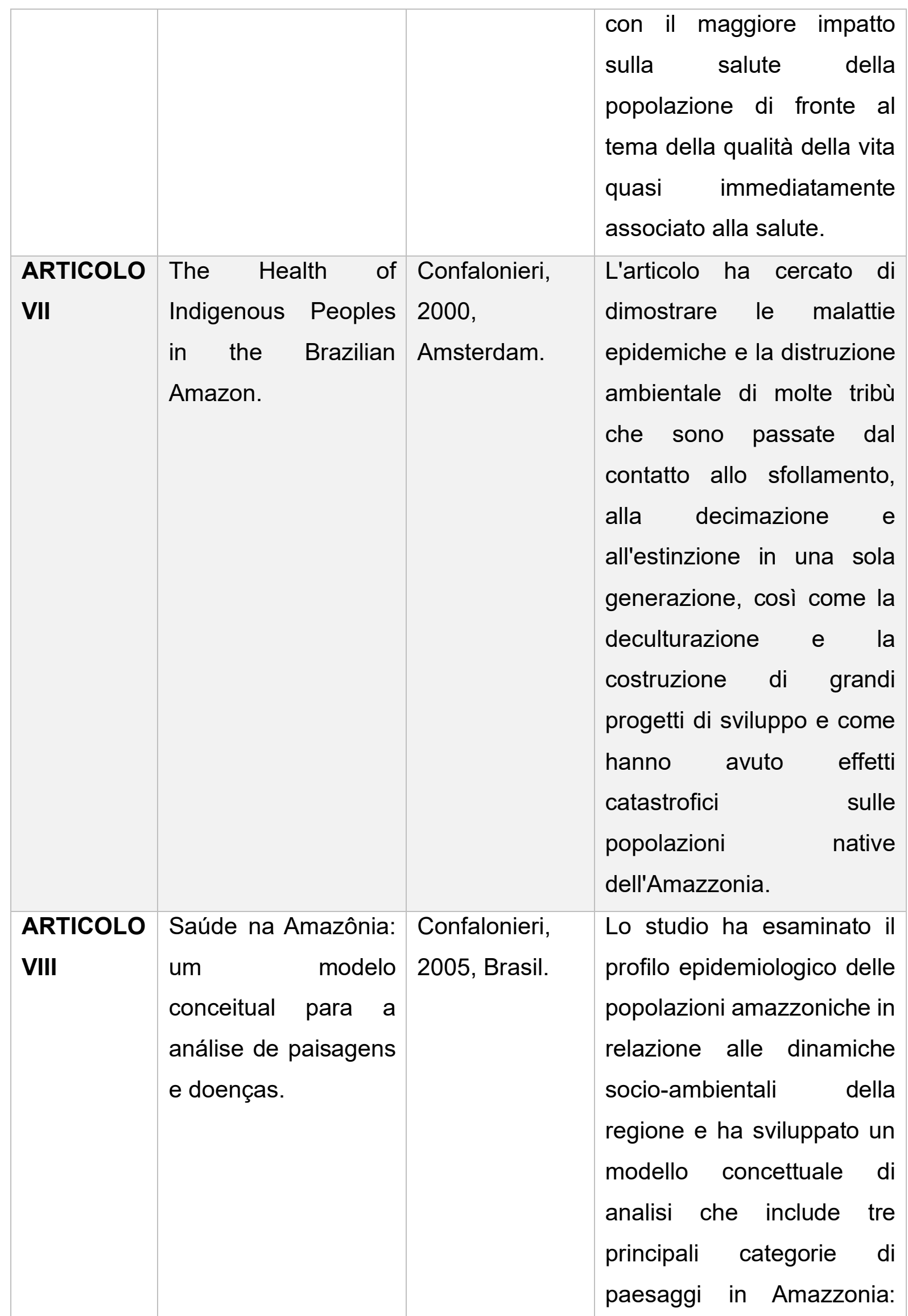




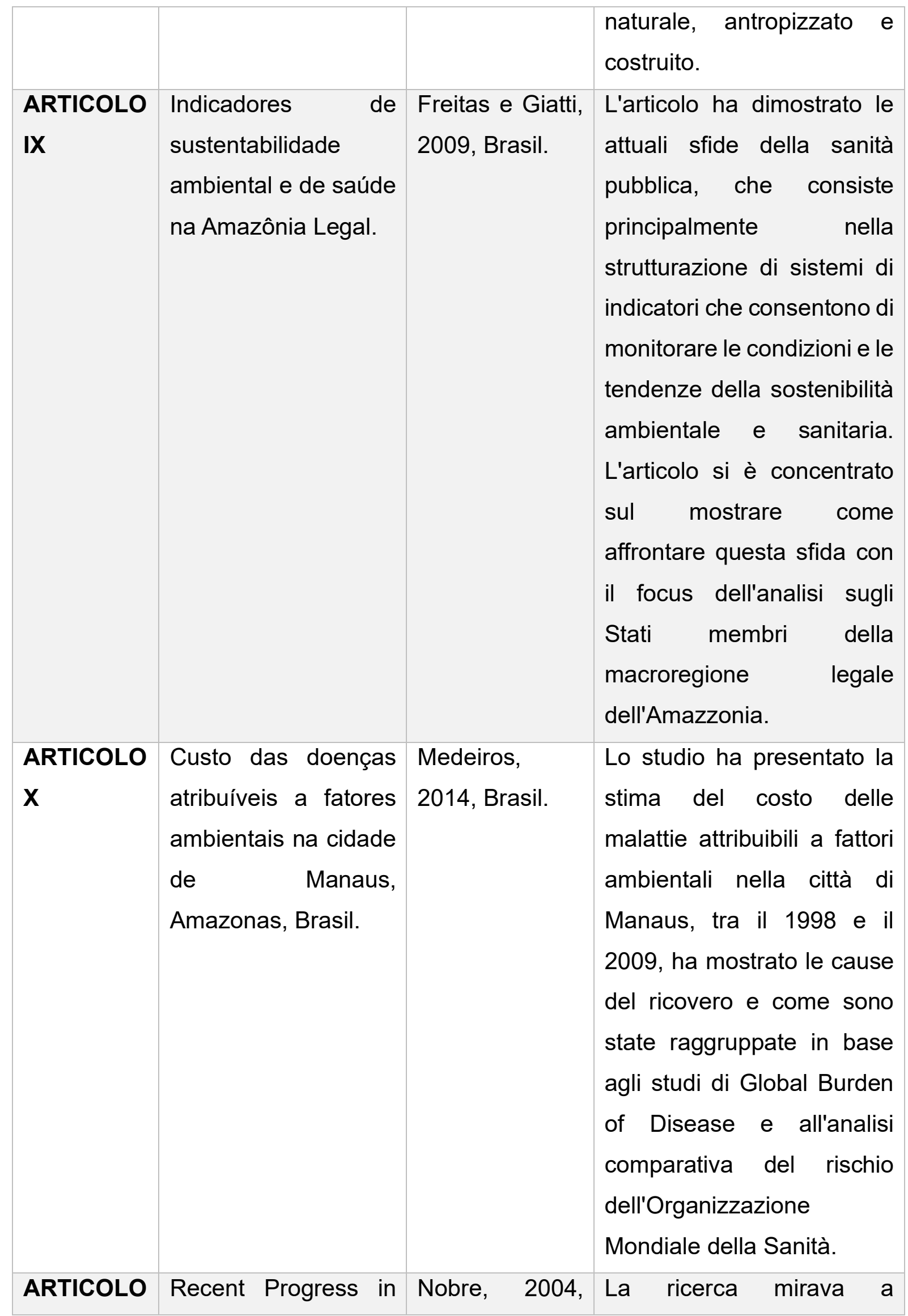

$\mathrm{RC}: 104517$

Link di accesso: https://www.nucleodoconhecimento.com.br/salute/comparsa-di-malattie 


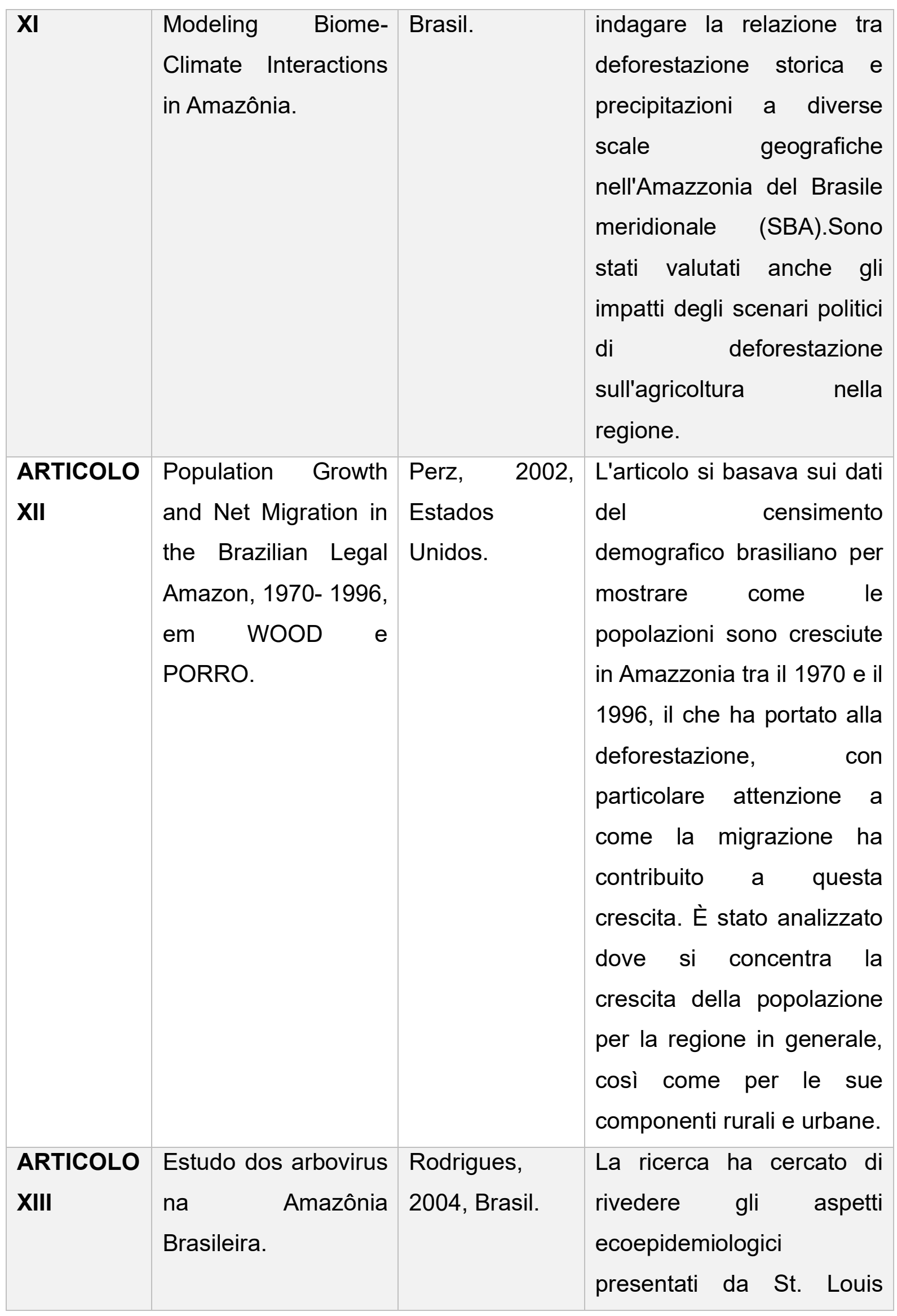




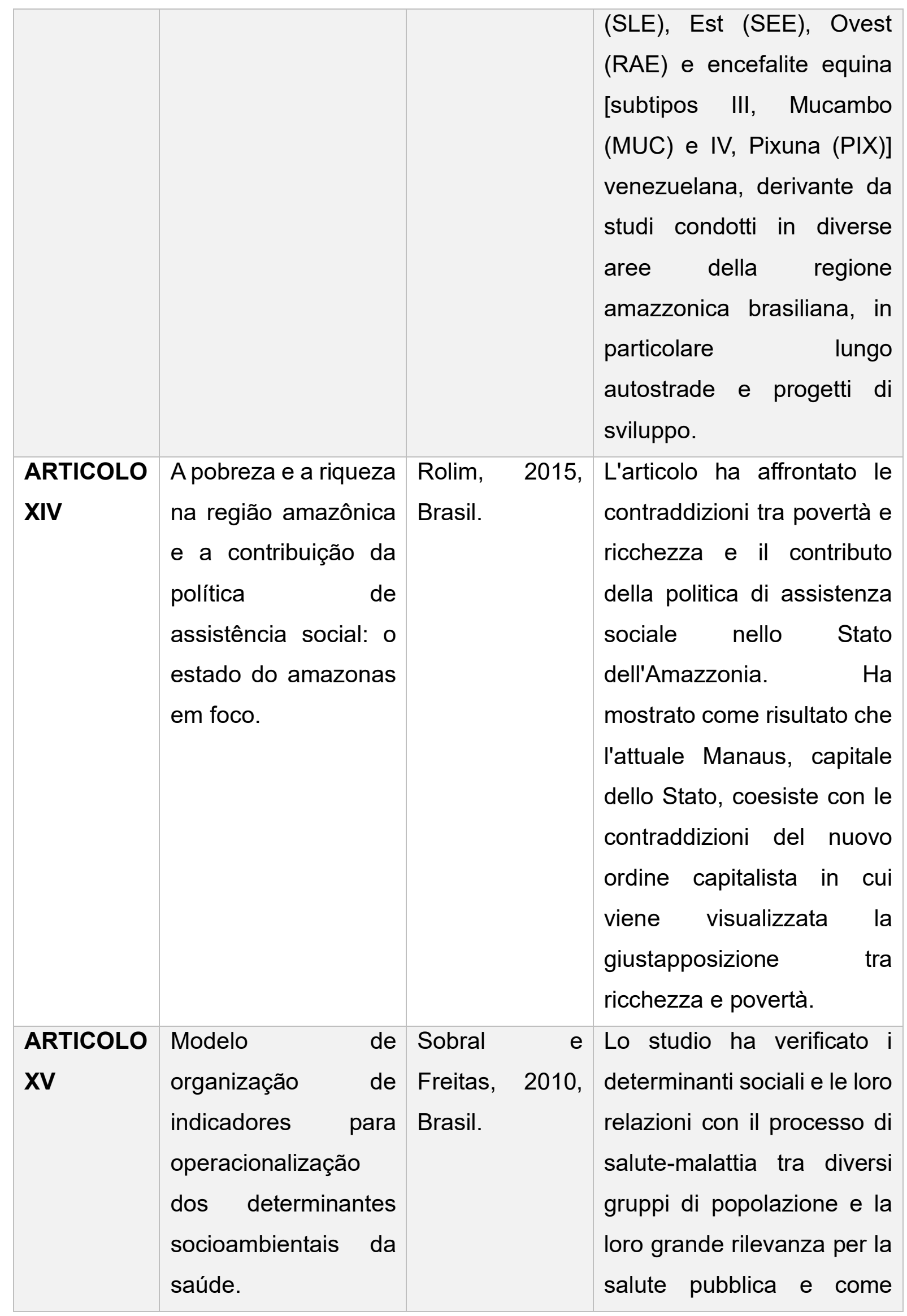

RC: 104517

Link di accesso: https://www.nucleodoconhecimento.com.br/salute/comparsa-di-malattie 


\begin{tabular}{|c|c|c|c|}
\hline & & & $\begin{array}{l}\text { stanno guadagnando } \\
\text { sempre più importanza. In } \\
\text { questo processo, sono stati } \\
\text { adottati modelli teorico- } \\
\text { concettuali per spiegare } \\
\text { come i determinanti sociali } \\
\text { generano disuguaglianze } \\
\text { sanitarie. }\end{array}$ \\
\hline $\begin{array}{l}\text { ARTICOLO } \\
\text { XVI }\end{array}$ & $\begin{array}{l}\text { Saúde ambiental e } \\
\text { desenvolvimento na } \\
\text { Amazônia legal: } \\
\text { indicadores } \\
\text { socioeconômicos, } \\
\text { ambientais } \\
\text { sanitários, desafios e } \\
\text { perspectivas. }\end{array}$ & $\begin{array}{l}\text { Viana et al., } \\
\text { 2016, Brasil. }\end{array}$ & $\begin{array}{l}\text { L'articolo ha cercato di } \\
\text { discutere i processi di } \\
\text { determinazione socio- } \\
\text { ambientale negli stati che } \\
\text { compongono l'Amazzonia } \\
\text { legale, sulla base } \\
\text { dell'analisi di indicatori } \\
\text { socioeconomici, ambientali } \\
\text { e sanitari. }\end{array}$ \\
\hline $\begin{array}{l}\text { ARTICOLO } \\
\text { XVII }\end{array}$ & $\begin{array}{l}\text { Saúde, ambiente e } \\
\text { desenvolvimento } \\
\text { econômico na } \\
\text { Amazônia. }\end{array}$ & $\begin{array}{l}\text { Vincentin } \\
\text { Minayo, 2003, } \\
\text { Brasil. }\end{array}$ & $\begin{array}{l}\text { Lo studio ha analizzato il } \\
\text { processo di trasformazione } \\
\text { del modello epidemiologico } \\
\text { di una popolazione } \\
\text { amazzonica costituita per } \\
\text { lavorare nello sviluppo di } \\
\text { Mineração Rio do Norte } \\
\text { S.A. (MRN), nel comune di } \\
\text { Oriximiná (PA), in località } \\
\text { Porto Trombetas. La } \\
\text { determinazione } \\
\text { processo salute-malattia è } \\
\text { stata sviluppata in base alle } \\
\text { particolari condizioni di } \\
\text { riproduzione sociale }\end{array}$ \\
\hline
\end{tabular}

RC: 104517

Link di accesso: https://www.nucleodoconhecimento.com.br/salute/comparsa-di-malattie 


\begin{tabular}{|c|c|c|c|}
\hline & & & $\begin{array}{l}\text { dell'impresa, ben diverse da } \\
\text { quelle delle popolazioni } \\
\text { legate al tradizionale } \\
\text { sviluppo socioeconomico } \\
\text { della regione. }\end{array}$ \\
\hline $\begin{array}{l}\text { ARTICOLO } \\
\text { XVIII }\end{array}$ & $\begin{array}{l}\text { Deforestation, } \\
\text { Hunting and the } \\
\text { Ecology of Microbial } \\
\text { Emergence }\end{array}$ & $\begin{array}{l}\text { Wolfe, } 2000, \\
\text { Estados } \\
\text { Unidos. }\end{array}$ & $\begin{array}{l}\text { Lo studio ha cercato di } \\
\text { capire come nuovi microbi } \\
\text { entrano nella popolazione } \\
\text { umana, quanto spesso } \\
\text { emergono i microbi } \\
\text { secondo la determinazione } \\
\text { data dalla diversità dei } \\
\text { microbi presenti } \\
\text { nell'ambiente, con anche il } \\
\text { livello di contatto tra una } \\
\text { potenziale diversità } \\
\text { microbica dell'ospite e la } \\
\text { suscettibilità del nuovo } \\
\text { ospite alle infezioni. }\end{array}$ \\
\hline
\end{tabular}

Fonte: autori propri.

Tra gli articoli scelti, la stragrande maggioranza degli studi ha dimostrato che i fattori ambientali e sociologici che contribuiscono all'emergere di malattie nella regione amazzonica dal 1970, come mostrato nell'articolo "Chagas disease and globalization of the Amazon" dell'autore Briceño-Léon (2007), rendendo questo bacino storico. Questo cambiamento nell'epidemiologia delle malattie nella regione amazzonica può essere spiegato dai cambiamenti ambientali e sociali negli ultimi 30 anni, come spiegato dall'autore.

Gli autori Browder e Godfrey (2017), Browder e Godfrey (2017), Cardoso (2015), Carrapato et al. (2017), Confalonieri (2000), Confalonieri (2005), Medeiros (2014), Nobre (2004), Perz (2002), Sobral e Freitas (2010) e Viana et al. (2016) nella sua RC: 104517

Link di accesso: https://www.nucleodoconhecimento.com.br/salute/comparsa-di-malattie 
ricerca pubblicata, in sintesi, ha mostrato la relazione dei determinanti sociali e ambientali e la loro connessione intrinseca con il processo salute-malattia di diverse popolazioni, la loro significativa rilevanza per la salute pubblica e il modo in cui sono diventati più importanti. In questo processo di anni di storie che l'Amazzonia ha affrontato con cambiamenti sociali e spaziali fisici e territoriali, e come conseguenza l'aggravamento delle malattie concentrate in questa regione.

\section{RASSEGNA DELLA LETTERATURA E DISCUSSIONE}

\subsection{ASPETTI REGIONALI}

Un fenomeno molto importante nell'ambiente amazzonico è l'interazione tra l'atmosfera e le foreste, che svolgono un ruolo importante nella produzione di precipitazioni nella regione. II clima è generalmente caldo e umido e la temperatura non cambia molto, essendo un importante regolatore dei processi biologici, in particolare quelli legati alle malattie infettive (NOBRE, 2004).

Un altro aspetto biofisico correlato riguarda la biodiversità, in particolare la diversità animale. Alcuni processi di infezione focale in quest'area, in particolare i processi di infezione virale, dipendono dagli insetti succhiatori di sangue (vettori) per la trasmissione (arbovirus). Maggiore è la diversità delle specie animali (vertebrati e invertebrati) in una determinata area, maggiore è il rischio di un nuovo processo di infezione da Wolfe (2000). Per esemplificare questo punto, circa 196 arbovirus sono stati identificati in Amazzonia, 32 dei quali possono infettare gli esseri umani (RODRIGUES, 2004).

Nobre (2004) sottolinea che in relazione alla società amazzonica, si possono evidenziare i seguenti importanti aspetti demografici:

lo. Bassa densità di popolazione regionale (circa 20 milioni di abitanti in un'area di 3,5 milioni di chilometri quadrati) (INPE, 2013; IBGE, 2013);

II. l'alto tasso di crescita fornito dall'immigrazione negli ultimi 30 anni. C'è anche una forte migrazione interregionale (PERZ, 2002);

RC: 104517

Link di accesso: https://www.nucleodoconhecimento.com.br/salute/comparsa-di-malattie 
III. le città sono altamente concentrate, soprattutto nelle capitali. Circa il 70\% della popolazione amazzonica vive in città grandi e piccole. L'emergere rapido, spontaneo e disordinato di molti di questi piccoli insediamenti urbani senza le necessarie infrastrutture igienico-sanitarie è caratteristico (BROWDER e GODFREY, 2017);

IV. c'è un gran numero di popolazioni tradizionali (indiani, ripari, spillatori di gomma, ecc.). che vivono di estrattivismo e piccola agricoltura (INPE, 2013; IBGE, 2013).

Un'altra importante caratteristica regionale riguarda le pratiche di utilizzo del suolo e il loro impatto ambientale e sociale. Alcune di queste pratiche producono cambiamenti ambientali che presentano rischi per la salute. È il caso dell'inquinamento da mercurio nelle miniere; a causa della mancanza di infrastrutture igienico-sanitarie nelle aree urbane, l'acqua è contaminata da microrganismi; il fumo da bruciare dopo la deforestazione causa problemi respiratori; cambiamenti locali nel ciclo idrologico che producono siti di riproduzione delle zanzare, per enumerare solo alcuni effetti più diretti (CONFALONIERI, 2000).

Va anche ricordato che i conflitti sociali, ad esempio, il possesso della terra portano alla violenza e ai conflitti culturali, e il drastico impatto delle popolazioni indigene isolate e l'introduzione di agenti microbici precedentemente sconosciuti da queste comunità, fornendo l'emergere di nuove malattie e aumentando il numero di casi di quelli esistenti nella regione (RODRIGUES, 2004).

Lo sfondo comune di questi eventi è che le politiche di sviluppo e utilizzo delle risorse ignorano il potenziale regionale, dimenticando la resilienza ambientale e la sostenibilità ecologica dell'Amazzonia, non portano alla cultura tradizionale o alla distribuzione del reddito e non si preoccupano dei rischi per la salute (CONFALONIERI, 2000).

In pratica, questo sta accadendo sia su iniziativa del governo, attraverso la realizzazione di grandi progetti infrastrutturali (autostrade, centrali idroelettriche, ecc.). e l'industria (estrazione mineraria commerciale), incoraggiando il bestiame (e la deforestazione), nonché attraverso attività estrattive spontanee come l'estrazione mineraria, la pesca, il disboscamento e altri (BRICEÑO-LEÓN, 2007; VICENTIN e MINAYO, 2003; FREITAS e GIATTI, 2009).

RC: 104517

Link di accesso: https://www.nucleodoconhecimento.com.br/salute/comparsa-di-malattie 


\subsection{INDICATORI SOCIO-AMBIENTALI}

Nello spazio che circonda le parti meridionali e orientali dell'Amazzonia (stati di Rondônia, Mato Grosso, Tocantins, Maranhão e Pará), negli ultimi anni si sono intensificati focolai di incendi, deforestazione accumulata e costruzione di autostrade, nonché aree di coltivazione e intensificazione del consumo di pesticidi (INPE, 2013; IBGE, 2013).

Queste risorse sono state chiamate da Nobre (2004) "arco di fuoco". Per quanto riguarda il consumo di pesticidi, i tassi raggiunti con queste sostanze nella regione sono stati espressivi, rappresentando il $18 \%$ del totale nazionale. Secondo i dati pubblicati nel 2009 dall'Istituto brasiliano di geografia e statistica (IBGE), lo stato del Mato Grosso ha raggiunto il più alto tasso nazionale di utilizzo di pesticidi per ettaro di superficie coltivata nell'Amazzonia legale (IBGE, 2013).

Secondo gli indicatori esaminati, i dati sull'aumento dei terreni coltivabili e dei pascoli sulle aree naturali e sulla deforestazione cumulativa in Amazzonia hanno raggiunto i tassi più alti nello stesso "arco di fuoco", con una chiara convergenza tra l'espansione dell'agricoltura estensiva e la deforestazione insieme al verificarsi di incendi e all'uso di pesticidi (CARDOSO, 2015).

Seguendo il ragionamento e il salvataggio di Confalonieri (2005), è possibile mostrare quanto le società amazzoniche incontrino difficoltà di accesso alla salute e, di conseguenza, mancanza di cure e malattie. L'autore richiama l'attenzione su fattori quali: bassa densità demografica regionale, crescita significativa delle migrazioni, comprese le migrazioni interregionali, alta concentrazione di città, soprattutto nelle capitali, con una mancanza di infrastrutture sanitarie e un gran numero di popolazioni tradizionali, danneggia i determinanti sociali in Amazzonia, causando gravi problemi di salute.

Per gli autori Medeiros et al. (2014) è stata inoltre discussa la situazione delle condizioni di lavoro nelle aree del paesaggio naturale e antropico dell'Amazzonia. Questo perché le condizioni e l'ambiente di lavoro sono determinanti delle malattie 
professionali e sono classificate come malattie non trasmissibili. Pertanto, la salute del lavoratore è concettualizzata come:

Um campo de saúde pública que tem como objeto de estudo e intervenção as relações produção-consumo e o processo saúde-doença. Neste campo, o trabalho é considerado como eixo organizador da vida social, espaço de dominação e resistência dos trabalhadores e determinantes das condições de vida e saúde das pessoas (BRASIL, 2018, p. 136).

Il lavoro è un fattore determinante importante per la vita e la salute delle persone. Sapendo che ci sono molte diverse attività, relazioni e condizioni di servizio nella regione amazzonica, è importante che gli operatori sanitari promuovano, prevengano e diagnostichino malattie legate alle attività che vengono eseguite nella regione amazzonica (CARDOSO, 2015).

In queste attività professionali molto specifiche dell'Amazzonia, che sono rare in altre parti del Brasile, si può menzionare: estrattivismo vegetale - professione, che comprende, tra gli altri, açaí estrattivo, compattatore di gomma, raccoglitore di noci del Brasile, piloti di barche da trasporto passeggeri, cercatori, pescatori e molti altri. Si percepisce anche che i rapporti di lavoro altamente degradanti, che in molti luoghi possono essere considerati analoghi alla schiavitù, sono abbastanza evidenti nella regione a causa della difficoltà di controllare la grande espansione della terra in Amazzonia.

Poiché si tratta di un'area ricca di minerali, i cercatori si stabiliscono in questa regione $\mathrm{e}$ in aree protette in cui l'estrazione mineraria è vietata. Come risultato dell'ingresso nelle aree forestali, sia i cercatori che gli estrattori di noci del Brasile e spillatori di gomma sono affetti da malattie batteriche trasmesse da vettori o protozoi non trasmissibili e protozoi che esistono nella foresta.

Tra questi, si può menzionare la malattia di Chagas, la malaria, la leishmaniosi e molte malattie virali nella regione. Pertanto, dato il gran numero di questi professionisti e i rischi che affrontano, il sistema sanitario dovrebbe dirigere le azioni in modo che le RC: 104517

Link di accesso: https://www.nucleodoconhecimento.com.br/salute/comparsa-di-malattie 
malattie direttamente o indirettamente correlate al lavoro non diventino un fattore di aumento della morbilità e della mortalità nella regione amazzonica (CARDOSO, 2015).

È importante sottolineare che il dibattito sul lavoro dell'Amazzonia e sulla sua portata è molto ricco, in quanto mostra anche che l'Amazzonia è un luogo che è ancora un recente di ricerca specifica e politiche di salute pubblica sulle condizioni di vita, le malattie sanitarie e il lavoro nella regione (BENSABATH e SOARES, 2016).

\subsection{INDICATORI EPIDEMIOLOGICI REGIONALI}

In relazione agli indicatori epidemiologici regionali, ci sono alcune importanti differenze tra la regione amazzonica e il resto del paese. Nel 1996 il tasso di ricoveri per malattie infettive e parassitarie era molto elevato $(12,7 \%)$, molto superiore alla media nazionale $(8,3 \%)$. Sebbene l'incidenza di queste lesioni sia diminuita in Amazzonia, il tasso di mortalità per queste malattie non è stato lo stesso (SUDAM, 2000).

Questa regione ospita la stragrande maggioranza dei casi di malaria nel paese (98\%), circa il 35\% dei casi di lebbra nel 2016 e la seconda più alta incidenza di tubercolosi tra tutte le regioni del paese. È anche un'area altamente endemica di epatite virale con frequenti epidemie, specialmente nell'Amazzonia occidentale (BENSABATH e SOARES, 2016).

È interessante notare che a causa delle condizioni geografiche della regione, così come il processo di urbanizzazione e la mancanza di servizi igienico-sanitari di base hanno contribuito al processo di malattie che sono riemerse in Amazzonia, come il colera nel 1991 e la dengue a metà degli anni 1990, oltre a questi, altre malattie causano anche un gran numero di morti nella popolazione della regione come la malaria, Leishmaniosi tegumentary infezioni parassitarie americane e soprattutto la tubercolosi (VIANA et al., 2016).

La letteratura rivela anche la relazione sproporzionata tra miglioramento della qualità della vita e degrado ambientale, cioè lo sviluppo avanza in modo insostenibile, e sottolinea l'importanza di considerare il costo delle malattie causate da fattori ambientali, che è aumentato nella regione amazzonica (MEDEIROS et al., 2014). RC: 104517

Link di accesso: https://www.nucleodoconhecimento.com.br/salute/comparsa-di-malattie 
Carrapato; Correa e Garcia (2017) hanno immediatamente collegato la qualità della vita alla salute. Gli autori sottolineano che, oltre ai modelli biomedici, ci sono alcuni fattori decisivi che hanno influenzato in modo decisivo o influenzeranno la salute personale. Pertanto, questi determinanti hanno attualmente un impatto maggiore sulla salute rispetto a quelli attualmente coperti dalla medicina. Hanno sottolineato l'esistenza di tre determinanti: ambiente, economia e società, per loro i determinanti sociali sono in gran parte responsabili dell'ingiustizia dell'accesso all'assistenza sanitaria.

\section{CONCLUSIONE}

Sulla base della domanda principale dello studio, è possibile dedurre che i principali fattori ambientali che contribuiscono all'emergere di malattie nella regione amazzonica sono la deforestazione, la combustione, l'inquinamento dei fiumi, il processo di urbanizzazione e l'agrobusiness, concludendo così che la discussione sullo studio ha finalmente identificato alcune questioni responsabili del numero di malattie in Amazzonia, dove si dovrebbero, ad esempio, considerare gli aspetti geografici, etnici, culturali e politici della salute nella regione amazzonica. Si denota che vi è una mancanza di politiche pubbliche che soddisfino la domanda di malattie sanitarie legate agli indicatori socio-ambientali della regione, nonché la necessità di condurre ulteriori studi e ricerche al fine di conoscere le avversità affrontate da queste popolazioni.

È anche da notare che i principali fattori ambientali che contribuiscono all'emergere di malattie nella regione amazzonica sono la deforestazione, la combustione, l'inquinamento dei fiumi, il processo di urbanizzazione e l'agrobusiness, cioè tutto dall'azione dell'uomo.

Tuttavia, lo studio mostra anche che dal punto di vista degli indicatori ambientali di salute e malattia, lo spazio amazzonico formato dall'interazione del sistema socioecologico ha caratteristiche distinte da altre parti del paese. Questa differenza è causata dal fondamento ecologico naturale e dalle sue forme di occupazione e sviluppo. 


\section{RIFERIMENTI}

BENSABATH, G. e SOARES, M. C. P. L'evoluzione delle conoscenze sull'epatite virale in Amazzonia: dall'epidemiologia e dall'eziologia alla prevenzione. Rev. Soc. Bras. Med. Trop., 37(supl. II):14-36, 2016.

BRASIL. Ministério da Saúde. Secretaria de Atenção à Saúde. Secretaria de Vigilância em Saúde. Saúde do trabalhador e da trabalhadora [recurso eletrônico]. Cadernos de Atenção Básica, n. 41 - Brasília: Ministério da Saúde, 2018. 136 p.: il.

BRICEÑO-LEÓN, R. Chagas disease and globalization of the Amazon. Cadernos de Saúde Pública, Rio de Janeiro, v. 23, n. 53, p. 33-40, 2007. Suplemento 1.

BROWDER, J. O. e GODFREY, B. J. Rainforest Cities. Urbanization, Development and Colonization of the Brazilian Amazon. Columbia University Press, New York, 2017, $429 \mathrm{pp}$.

CAÑETE, T. M. R..; RAVENA-CAÑETE, V. Populações Tradicionais Amazônicas: revisando conceitos. In: V Encontro Nacional da Associação Nacional de PósGraduação e Pesquisa em Ambiente e Sociedade, 2010, Florianópolis Anais, V Encontro Nacional da Associação Nacional de Pós-Graduação e Pesquisa em Ambiente e Sociedade, 2010.

CARDOSO, A. C. M. O trabalho como determinante do processo saúde-doença. Tempo soc., São Paulo, v. 27, n. 1, p. 73-93, junho de 2015.

CARRAPATO, P.; CORREIA, P.; GARCIA, B. Determinante da saúde no Brasil: a procura da equidade na saúde. Saúde soc., São Paulo, v. 26, n. 3, p. 676-689, Sept. 2017.

CONFALONIERI, U. E. C. (org.). The Health of Indigenous Peoples in the Brazilian Amazon. Background paper for the World Bank. Royal Tropical Institute, Amsterdam, 2000, $218 \mathrm{pp}$. 
CONFALONIERI, U. E. C. Saúde na Amazônia: um modelo conceitual para a análise de paisagens e doenças. Estud. av., São Paulo, v. 19, n. 53, p. 221-236, Apr. 2005.

FREITAS, C. M.; GIATTI, L. L. Indicadores de sustentabilidade ambiental e de saúde na Amazônia Legal. Cadernos de Saúde Pública, Rio de Janeiro, v. 25, n. 6, p. 1251-1266, 2009.

IBGE - INSTITUTO BRASILEIRO DE GEOGRAFIA E ESTATíSTICA. Contas nacionais: 2011. Rio de Janeiro, RJ, 2013.

INPE - Instituto Nacional De Pesquisas Espaciais. Monitoramento da floresta amazônica brasileira por satélite: 1988/2011. 2013.

MEDEIROS, M. S. de et al. Custo das doenças atribuíveis a fatores ambientais na cidade de Manaus, Amazonas, Brasil. Ciênc. Saúde coletiva [online]. 2014, vol.19, n.2, pp.599-608. 2014.

NOBRE, C. A. Recent Progress in Modeling Biome-Climate Interactions in Amazônia. Resumo 19.2. Anais III Conf. Cient.do LBA, 27-29 de julho, 2004. Brasília, DF, Cd-Rom.

PERZ, S. Population Growth and Net Migration in the Brazilian Legal Amazon, 1970- 1996, em WOOD e PORRO. Deforestation and Land Use in the Amazon. Gainesville, University Press of Florida, 2002, pp. 95-106.

RODRIGUES, S. G. Estudo dos arbovirus na Amazônia Brasileira. Em: Resumos do III Simpósio Internacional sobre Arbovirus nos Trópicos e Febres Hemorrágicas, Belém, Pará, 30/11 - 3/12, 2004. Instituto Evandro Chagas, 2004.

ROLIM, D. C. A pobreza e a riqueza na região amazônica e a contribuição da política de assistência social: o estado do amazonas em foco. VII Jornada Internacional de Políticas Públicas. Programa de Pós-graduação em políticas públicas. Universidade Federal do Maranhão, 2015. 
SOBRAL, A.; FREITAS, C. M. Modelo de organização de indicadores para operacionalização dos determinantes socioambientais da saúde. Saúde e Sociedade, São Paulo, v. 19, n. 1, p. 35-47. 2010.

SUDAM. Diagnóstico e cenarização macrossocial da Amazônia legal: perfil da saúde na Amazônia legal e o contexto brasileiro. Belém, Pnud, 2000, 54 p.

VIANA, Rosana Lima; FREITAS, Carlos Machado de; GIATTI, Leandro Luiz. Saúde ambiental e desenvolvimento na Amazônia legal: indicadores socioeconômicos, ambientais e sanitários, desafios e perspectivas. Saúde e Sociedade, v. 25, p. 233246, 2016.

VICENTIN, G.; MINAYO, C. G. Salute, ambiente e sviluppo economico in Amazzonia. Scienza e salute pubblica, Rio de Janeiro, v. 8, n. 4, p. 1069-1085, 2003. WOLFE, N. Deforestazione, caccia ed ecologia dell'emergenza microbica. Cambiamento globale \& Um. Salute, 1(1):10-25, 2000.

Inviato: Dicembre, 2021.

Approvato: Dicembre 2021. 